\title{
Tratamento da doença hemorroidária com ligadura elástica: estudo prospectivo com 59 pacientes
}

\author{
Rubber band ligation for the treatment of hemorrhoidal disease: prospective study \\ with 59 patients
}

\author{
MARCEL MACHADO DA MOTTA ${ }^{1}$, JOSÉ BATISTA DA SILVA JÚNIOR ${ }^{1}$, LARICE OLIVEIRA SANTANA ${ }^{1}$, \\ IGOR LIMA FERDANDES ${ }^{1}$, ALEX RODRIGUES MOURA ${ }^{2}$, ANA CAROLINA LISBOA PRUDENTE \\ JUVENAL DA ROCHA TORRES NETO ${ }^{4}$
}

${ }^{1}$ Acadêmicos de Medicina da Universidade Federal de Sergipe (UFS) - Aracaju (SE), Brasil. ${ }^{2}$ Médico Residente de Coloproctologia do Hospital Universitário da UFS - Aracaju (SE), Brasil. ${ }^{3}$ Médica Coloproctologista do Hospital Universitário da UFS - Aracaju (SE), Brasil. ${ }^{4}$ Chefe do Serviço de Coloproctologia do Hospital

Universitário da UFS - Aracaju (SE), Brasil.

MOTTA MM, JÚNIOR JBS, SANTANA LO, FERDANDES IL, MOURA AR, PRUDENTE ACL, NETO JRT. Tratamento da doença hemorroidária com ligadura elástica: estudo prospectivo com 59 pacientes. Rev bras Coloproct, 2011;31(2): 139-146.

RESUMO: A ligadura elástica (LE) é considerada um método minimamente invasivo para o tratamento da doença hemorroidária (DH) e apresenta vantagens em relação à hemorroidectomia, como: simplicidade de execução e realização ambulatorial sem o emprego de anestesia. Trata-se de um método eficaz, principalmente nos portadores de DH grau II. Porém, apresenta complicações, sendo as mais frequentes: dor, tenesmo, hematoquezia e retenção urinária. Alguns trabalhos mostram complicações mais graves, como sangramentos vultosos que necessitaram de hemotransfusão. Assim, este trabalho avaliou a eficácia e a morbidade do tratamento da DH pelo método da LE. Foi um estudo prospectivo, com 59 pacientes. Destes, cinco (8,5\%) eram portadores de DH grau I, 33 (55,9\%) de DH grau II e $21(35,6 \%)$ de DH grau III. Todos os pacientes foram submetidos pelo menos a duas sessões. Nas 135 sessões realizadas, encontramos: hematoquezia em $62(45,9 \%)$, dor intensa em $39(28,9 \%)$, sintomas vagais em 10 (7,4\%) e pseudoestrangulamento em um (0,7\%) sessão. A taxa de cura do prolapso hemorroidário entre os portadores de DH grau II foi de 87,9\% e entre aqueles com DH grau III, de 76,2\%. O tratamento da DH pelo método da ligadura elástica mostrou-se seguro e com boa taxa de cura.

Palavras-chave: hemorroidas; ligadura elástica; complicações.

\section{INTRODUÇÃO}

Atualmente, várias são as opções terapêuticas disponíveis para o tratamento da doença hemorroidária $(\mathrm{DH})$, variando desde uma simples mudança nos hábitos alimentares até as mais diferentes técnicas cirúrgicas de excisão dos mamilos hemorroidários doentes. A fotocoagulação, a crioterapia, a escleroterapia, a diatermia e a eletrocoagulação são considerados tratamentos conservadores do tipo invasivo. Tais métodos não cirúrgicos induzem a uma fibrose tecidual com posterior fixação do coxim hemorroidário. Tam- bém incluída nesse último grupo está a técnica da ligadura elástica $(\mathrm{LE})^{1,2}$.

A técnica da LE foi primeiro descrita por Blaisdell (1958), mas só tornou-se popular após Barron (1964), em Detroit, aperfeiçoá-la com um aparelho modificado. Dentre os benefícios da LE, podemos destacar: simplicidade de execução da técnica, baixo custo, possibilidade ser realizada ambulatorialmente e sem o emprego de anestesia. Além disso, a LE apresenta resultados tão bons quanto os da hemorroidectomia, quando são tratadas hemorroidas de segundo grau ${ }^{3}$. Índices de cura maiores que $70 \%$ em

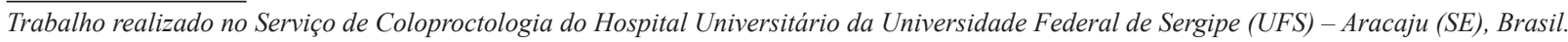


hemorroidas do terceiro grau também são vistos em diversos trabalhos ${ }^{4}$. Esses dados, aliados a níveis de comorbidades muito mais brandos que os das cirurgias de hemorroidectomia, fazem com que a LE seja a primeira opção de tratamento da doença hemorroidária até a classificação de terceiro grau na maioria dos casos 5 .

Dentre as complicações citadas na literatura após a realização da LE, encontram-se: dor, retorragias, sintomas vagais, fissura anal, fístula anal e abscesso perianal $^{6}$. A dor é, sem dúvida, a complicação mais comum. Apesar de ser, geralmente, de fraca intensidade, dores de forte intensidade variam de 5,8 a 40,0\% nos casos relatados na literatura ${ }^{5,7}$.

Assim, o objetivo deste trabalho foi avaliar o tratamento da doença hemorroidária pelo método da ligadura elástica quanto a sua morbidade e eficácia.

\section{PACIENTES E MÉTODOS}

\section{Casuística}

Foram acompanhados os pacientes submetidos ao tratamento da doença hemorroidária pelo método da ligadura elástica no Hospital Universitário da Universidade Federal de Sergipe (HU/UFS) no período de agosto de 2009 a julho de 2010.

\section{Critérios de inclusão}

Foram incluídos os pacientes tratados pelo método da ligadura elástica que compareceram a todas as sessões.

\section{Critérios de exclusão}

Foram excluídos pacientes com outras doenças anorretais associadas (fissura anal e fístula anal).

\section{Método}

\section{Realização da ligadura elástica e coleta de dados}

Pacientes atendidos no ambulatório de coloproctologia do HU/UFS, portadores de hemorroidas internas de I, II ou III graus, sem componente externo importante ou outras doenças anorretais associadas (fissura anal e fístula anal), foram encaminhados para o tratamento com ligadura elástica.

Para a sua realização, utilizou-se pinça de colocação de anel elástico por aspiração Ferrari Medical ${ }^{\circledR}$, sendo realizada a ligadura de um ou dois mamilos por sessão. Todos os pacientes tiveram pelo menos três mamilos ligados ao final do tratamento. O intervalo entre cada sessão foi de uma semana. Além disso, foi marcada uma consulta de retorno 30 dias após a realização da última sessão.

O procedimento foi realizado em decúbito lateral esquerdo (Sims). A analgesia foi realizada com Lisador $^{\circledR}$ (dipirona sódica, cloridrato de prometazina e cloridrato de adifenina) comprimido, da seguinte maneira: um comprimido 30 minutos antes da realização do procedimento, com a manutenção de seis em seis horas até que a dor cessasse.

Antes do início do tratamento, os pacientes que aceitaram participar da pesquisa e assinaram o Termo de Consentimento Livre e Esclarecido foram questionados a respeito das queixas clínicas que os levaram a procurar o serviço de coloproctologia do HU/UFS. A gradação da doença hemorroidária foi feita novamente no dia da realização da primeira sessão por meio da anuscopia.

A morbidade de cada sessão foi avaliada na sessão subsequente ou na consulta de retorno. A dor foi avaliada em uma escala de zero a dez, sendo interpretada da seguinte forma: nota zero foi considerada como ausência de dor; notas de um a três foram consideradas dor leve; notas de quatro a sete foram consideradas dor moderada; notas de oito a dez foram consideradas dor intensa. $\mathrm{O}$ tempo transcorrido até que a dor cessasse também foi questionado.

Trinta dias após a última sessão, na consulta de retorno, os pacientes foram questionados acerca dos sinais e sintomas apresentados nos dias logo após a última sessão, bem como sobre o seu quadro clínico atual. A eficácia do tratamento foi avaliada por meio da anuscopia: caso, após 30 dias da última sessão, não houvesse mamilo residual ou prolapso mucoso, o tratamento seria considerado eficaz e o paciente considerado curado da doença hemorroidária; na persistência de mamilo hemorroidário ou prolapso mucoso, o tratamento seria considerado ineficaz e o paciente seria encaminhado à ligadura do mamilo residual ou à hemorroidectomia.

Além disso, ao final da consulta de retorno, todos os pacientes responderam a duas perguntas. A primeira questionava o grau de satisfação com o tratamento (questionamos se eles consideravam-se 
satisfeitos, parcialmente satisfeitos ou insatisfeitos com o tratamento). A segunda pergunta foi a seguinte: "Caso fosse necessário, o(a) senhor(a) realizaria novamente o tratamento?".

\section{Variáveis estudadas}

Dados demográficos (idade, gênero e procedência), quadro clínico (antes e depois do tratamento), gradação da doença hemorroidária, morbidade e eficácia do tratamento instituído.

\section{Considerações éticas}

O projeto foi aprovado pelo Comitê de Ética e Pesquisa do Hospital Universitário da Universidade Federal de Sergipe (protocolo n ${ }^{\circ}$ 0049,0,107,000-09). Todos os pacientes foram esclarecidos quanto aos objetivos da pesquisa e assinaram Termo de Consentimento Livre e Esclarecido. Esta pesquisa não ofereceu riscos nem gastos adicionais ao sujeito da pesquisa. $\mathrm{O}$ anonimato do entrevistado foi respeitado.

\section{Análise estatística}

Os dados coletados foram lançados em um sistema de banco de dados informatizado e, posteriormente, foram submetidos à análise estatística, utilizandose o software Statistics Package of the Social Science (SPSS/PC+) versão 9.0 (1998). Para a caracterização da amostra, foram utilizadas tabelas de frequência simples, bem como a determinação de mediana, média e desvio-padrão.

\section{RESULTADOS}

Foram incluídos na pesquisa 59 pacientes. Destes, $25(42,4 \%)$ foram do gênero masculino e $34(57,6 \%)$ do feminino. A idade variou entre 19 e 70 anos, com uma média de 45,08 anos e desvio-padrão de 13,36. Vinte e quatro $(40,7 \%)$ pacientes procederam da capital e $35(59,3 \%)$ do interior.

No quadro clínico inicial, dor anal estava presente em $40(67,8 \%)$ pacientes, prurido anal em 35 $(59,3 \%)$, hematoquezia em $30(50,8 \%)$, soiling em 18 $(30,5 \%)$ e prolapso da mucosa anal ao ato de defecar em 31 (52,5\%). Ardor anal durante a evacuação estava presente em $10(16,9 \%)$ pacientes e $25(42,4 \%)$ relataram ardor após a evacuação. Além disso, 30 (50,8\%) pacientes eram constipados crônicos. Ao exame proc- tológico, encontramos: cinco $(8,5 \%)$ pacientes com DH grau I, 33 (55,9\%) pacientes com DH grau II e 21 $(35,6 \%)$ pacientes com DH grau III.

Um total de 207 ligaduras foram realizadas em 135 sessões. Todos os pacientes realizaram pelo menos duas sessões. Assim, 49 (83,1\%) pacientes necessitaram de duas sessões, cinco $(8,5 \%)$ de três sessões, quatro $(6,8 \%)$ pacientes de quatro sessões e um $(1,7 \%)$ de seis sessões. Na primeira sessão, $52(88,1 \%)$ pacientes tiveram dois mamilos ligados e sete $(11,9 \%)$ apenas um. Já na segunda sessão, 45 (76,3\%) pacientes tiveram um mamilo ligado e $14(23,7 \%)$ tiveram dois mamilos ligados.

Após a primeira sessão, ocorreram as seguintes complicações: $12(20,3 \%)$ pacientes relataram dor leve, 17 (28,8\%) dor moderada, 24 (40,7\%) dor intensa e seis $(10,2 \%)$ não relataram dor. Dos pacientes que relataram dor, $20(37,7 \%)$ afirmaram que a dor durou menos de 24 horas, 11 (20,7\%) relataram uma duração entre 24 e 48 horas, e em 22 (41,6\%) pacientes a dor demorou mais que 48 horas para cessar. Dos setes pacientes que foram submetidos à ligadura de apenas um mamilo na primeira sessão, nenhum relatou dor intensa, um $(14,3 \%)$ relatou dor moderada e dois $(28,6 \%)$ não relataram dor. Já nos outros 52 pacientes que foram submetidos à ligadura de dois mamilos, $24(46,1 \%)$ relataram dor intensa, $16(30,8 \%)$ dor moderada e quatro $(7,7 \%)$ não relataram dor. Outras queixas após a primeira sessão foram: $34(57,6 \%)$ pacientes relataram hematoquezia, $14(23,7 \%)$ dor ao evacuar, $13(22,0 \%)$ tenesmo, sete $(11,9 \%)$ sintomas vagais, sete $(11,9 \%)$ tenesmo vesical, seis $(10,2 \%)$ observaram a saída de secreção purulenta pelo ânus e três $(5,1 \%)$ a saída de secreção hialina. Além disso, 47 $(79,7 \%)$ pacientes precisaram afastar-se de suas atividades laborais apenas no dia em que a primeira sessão foi realizada.

Após a segunda sessão, ocorreram as seguintes complicações: $17(28,8 \%)$ pacientes relataram dor leve, $16(27,1 \%)$ dor moderada, $12(20,3 \%)$ dor intensa e 14 $(23,7 \%)$ não relataram dor. Dos pacientes que relataram dor, $23(51,1 \%)$ afirmaram que a dor durou menos de 24 horas, sete $(15,6 \%)$ relataram uma duração entre 24 e 48 horas, e em 15 (33,3\%) pacientes a dor demorou mais que 48 horas para cessar. Dos 45 pacientes que foram submetidos à ligadura de apenas um mamilo na segunda sessão, $11(24,4 \%)$ relataram dor intensa, 
$12(26,6 \%)$ dor moderada e $11(24,4 \%)$ não relataram dor. Já nos outros 14 pacientes que foram submetidos à ligadura de dois mamilos, um $(7,1 \%)$ relatou dor intensa, quatro $(28,6 \%)$ dor moderada e três $(21,4 \%)$ não relataram dor. Outras queixas após a segunda sessão foram: $21(35,6 \%)$ pacientes relataram hematoquezia, $12(20,3 \%)$ dor ao evacuar, dez $(16,9 \%)$ tenesmo, cinco $(8,5 \%)$ tenesmo vesical, seis $(10,2 \%)$ observaram a saída de secreção purulenta pelo ânus, três $(5,1 \%)$ a saída de secreção hialina e dois $(3,4 \%)$ sintomas vagais. Além disso, $51(86,4 \%)$ pacientes precisaram afastarse de suas atividades laborais apenas no dia em que a segunda sessão foi realizada.

Nos dez pacientes que realizaram uma terceira sessão, encontramos as seguintes complicações: cinco $(50,0 \%)$ relataram dor leve, um $(10,0 \%)$ dor moderada, dois $(20,0 \%)$ dor intensa e dois $(20,0 \%)$ não relataram dor. Dos pacientes que relataram dor, seis $(75,0 \%)$ afirmaram que a dor durou menos de 24 horas, um (12,5\%) relatou uma duração entre 24 e 28 horas, e em um $(12,5 \%)$ paciente a dor demorou mais que 48 horas para cessar. Outras queixas após a terceira sessão foram: cinco $(50,0 \%)$ pacientes relataram hematoquezia, três $(30,0 \%)$ dor ao evacuar, dois $(20,0 \%)$ tenesmo, dois $(20,0 \%)$ tenesmo vesical, um $(10,0 \%)$ sintomas vagais e um $(10,0 \%)$ observou a saída de secreção purulenta pelo ânus. Além disso, oito $(80,0 \%)$ pacientes precisaram afastar-se de suas atividades laborais apenas no dia em que a terceira sessão foi realizada.

Nos cinco pacientes que realizaram uma quarta sessão, encontramos as seguintes complicações: um $(20,0 \%)$ paciente relatou dor leve, dois $(40,0 \%)$ dor moderada, um $(20,0 \%)$ dor intensa e um $(20,0 \%)$ não relatou dor. Dois $(40,0 \%)$ pacientes relataram tenesmo, um $(20,0 \%)$ tenesmo vesical e dois $(40,0 \%)$ hematoquezia. Nenhum paciente relatou sintomas vagais, dor ao evacuar ou presença de secreção anal purulenta. Além disso, quatro $(80,0 \%)$ pacientes precisaram afastar-se de suas atividades laborais apenas no dia em que a quarta sessão foi realizada.

Nas 135 sessões realizadas, encontramos: dor leve em 35 (25,9\%) sessões, moderada em 38 $(28,1 \%)$, intensa em $39(28,9 \%)$, e em $23(17,1 \%)$ sessões não houve relato de dor. Nas sessões em que houve relato de dor, em $50(44,6 \%)$ ela durou menos que 24 horas, em 19 (17,0\%) durou entre 24 e 48 ho- ras, e em $43(38,4 \%)$ durou mais que 48 horas. Também encontramos: sintomas vagais em dez $(7,4 \%)$ sessões, hematoquezia em $62(45,9 \%)$, dor ao evacuar em 29 (21,5\%), tenesmo em 27 (20,0\%), tenesmo vesical em $15(11,1 \%)$, secreção anal purulenta em 13 (9,6\%), secreção anal hialina em seis $(4,4 \%)$ e pseudoestrangulamento em uma $(0,7 \%)$ sessão. Além disso, em $112(83,0 \%)$ sessões, os pacientes necessitaram afastar-se de suas atividades laborais apenas no dia em que a sessão foi realizada. O tempo médio de afastamento das atividades laborais foi de 1,6 dias por sessão (Tabela 1).

Quarenta pacientes realizaram duas ligaduras na primeira sessão e uma na segunda. Assim, entre esses pacientes, encontramos a seguinte distribuição da dor: na primeira sessão, seis $(15 \%)$ pacientes relataram dor leve, $11(27,5 \%)$ dor moderada, $20(50 \%)$ dor intensa e três $(7,5 \%)$ não tiveram dor; na segunda sessão, 11 $(27,5 \%)$ relataram dor leve, $11(27,5 \%)$ dor moderada, nove $(22,5 \%)$ dor intensa e nove $(22,5 \%)$ não tiveram dor. Naqueles que relataram dor (37 na primeira sessão e 31 na segunda), observamos: na primeira sessão, $11(29,7 \%)$ pacientes afirmaram que a dor cessou em menos de 24 horas, em dez $(27,0 \%)$ a dor levou de 24 a 48 horas para cessar e em $16(43,2 \%)$ durou mais que 48 horas; na segunda sessão, $17(54,8 \%)$ pacientes afirmaram que a dor cessou em menos de 24 horas, em cinco $(16,1 \%)$ a dor levou de 24 a 48 horas para cessar e em nove $(29,0 \%)$ durou mais que 48 horas.

Ainda neste grupo de pacientes que realizaram duas ligaduras na primeira sessão e uma na segunda, encontramos as seguintes complicações: na primeira sessão, quatro $(10 \%)$ pacientes relataram sintomas vagais, dez (25\%) tenesmo, 11 (27,5\%) dor ao evacuar, seis $(15 \%)$ retenção urinária, $23(57,5 \%)$ hematoquezia, cinco (12,5\%) secreção anal purulenta, um $(2,5 \%)$ secreção anal hialina e $11(27,5 \%)$ necessitaram afastar-se de suas atividades laborais por pelo menos um dia além daquele em que a sessão foi realizada; na segunda sessão, um $(2,5 \%)$ paciente relatou sintomas vagais, oito $(20,0 \%)$ relataram tenesmo, $10(25,0 \%)$ dor ao evacuar, três $(7,5 \%)$ retenção urinária, $14(35,0 \%)$ hematoquezia, cinco (12,5\%) secreção anal purulenta, dois $(5,0 \%)$ secreção anal hialina e sete $(17,5 \%)$ necessitaram afastar-se de suas atividades laborais por pelo menos um dia além daquele em que a sessão foi realizada (Tabela 2). 
Rev bras Coloproct Abril/Junho, 2011
Tratamento da doença hemorroidária com ligadura elástica:

estudo prospectivo com 59 pacientes

Marcel Machado Da Motta e Cols.
Vol. 31

$\mathbf{N}^{\circ} 2$
Na consulta de retorno, 30 dias após a última sessão, encontramos o seguinte quadro clínico: dor anal estava presente em cinco $(8,5 \%)$ pacientes, prurido anal em 10 (16,9\%), hematoquezia em dois (3,4\%), soiling em quatro $(6,8 \%)$ e prolapso da mucosa anal ao ato de defecar em dois (3,4\%). Ardor anal durante a evacuação estava presente em um $(1,7 \%)$ paciente, e dez $(16,9 \%)$ pacientes relataram ardor após a evacuação. Além disso, $11(18,6 \%)$ pacientes relataram constipação. Na Tabela 3 , encontramos a comparação do quadro clínico antes e depois do tratamento. Ao exame proctológico após o tratamento, encontramos: $48(81,4 \%)$ pacientes com cura do prolapso, dois $(3,4 \%)$ pacientes com $\mathrm{DH}$ grau I, oito $(13,6 \%)$ pacientes com DH grau II e um $(1,7 \%)$ paciente com DH grau III. Dos 33 pacientes com DH grau II antes do tratamento, 29 (87,9\%) apresentaram cura do prolapso à anuscopia. Já dos 21 pacientes com DH grau III, 16 (76,2\%) não apresentavam prolapso da mucosa anal à anuscopia (Tabela 4).

Ao serem questionados sobre a satisfação com o tratamento, 40 pacientes $(67,8 \%)$ afirmaram estar completamente satisfeitos com o tratamento, 17 pacientes $(28,8 \%)$ estavam parcialmente satisfeitos e dois $(3,4 \%)$ pacientes diziam-se insatisfeitos com o tratamento (Gráfico 1). Quando questionados se realizariam nova- mente o tratamento, caso fosse necessário, 58 (98,3\%) pacientes responderam que sim e um paciente $(1,7 \%)$ disse que não faria novamente o tratamento.

\section{DISCUSSÃO}

As hemorroidas são coxins de tecido conjuntivo fibroelástico, ricos em plexos vasculares situados na submucosa da região anorretal, importantes na oclu-

Tabela 1. Complicações observadas após a ligadura elástica em todas as 135 sessões.

\begin{tabular}{lc}
\hline $\begin{array}{l}\text { Complicações após o procedimento } \\
\text { (em todas as 135 sessões) }\end{array}$ \\
\hline Dor modera/intensa & $77(57,0 \%)$ \\
Hematoquezia & $62(45,9 \%)$ \\
Dor ao evacuar & $29(21,5 \%)$ \\
Tenesmo retal & $27(20,0 \%)$ \\
Tenesmo vesical & $15(11,1 \%)$ \\
Secreção anal purulenta & $13(9,6 \%)$ \\
Sintomas vagais & $10(7,4 \%)$ \\
Secreção anal hialina & $6(4,4 \%)$ \\
Pseudoestrangulamento & $1(0,7 \%)$ \\
Absentismo laboral maior que um dia & $23(17,0 \%)$ \\
\hline
\end{tabular}

Tabela 2. Complicações nos 40 pacientes que realizaram duas ligaduras na primeira sessão e uma ligadura na segunda sessão.

\begin{tabular}{lcc}
\hline Complicações após o procedimento & $\mathbf{1}^{\mathbf{a}}$ Sessão & $\mathbf{2}^{\mathbf{a}}$ Sessão \\
\hline Dor modera/intensa & $31(77,5 \%)$ & $20(50,0 \%)$ \\
Hematoquezia & $23(57,5 \%)$ & $14(35,0 \%)$ \\
Dor ao evacuar & $11(27,5 \%)$ & $10(25,0 \%)$ \\
Tenesmo retal & $10(25,0 \%)$ & $8(20,0 \%)$ \\
Tenesmo vesical & $6(15,0 \%)$ & $3(7,5 \%)$ \\
Sintomas vagais & $4(10,0 \%)$ & $1(2,5 \%)$ \\
Absentismo laboral maior que um dia & $11(27,5 \%)$ & $7(17,5 \%)$ \\
\hline
\end{tabular}

Tabela 3. Comparação do quadro clínico antes e depois do tratamento.

\begin{tabular}{lcc}
\hline Apresentação clínica & Antes do tratamento & Após o tratamento \\
\hline Dor anal & $40(67,8 \%)$ & $5(8,5 \%)$ \\
Prurido anal & $35(59,3 \%)$ & $10(16,9 \%)$ \\
Prolapso & $31(53,5 \%)$ & $2(3,4 \%)$ \\
Hematoquezia & $30(50,8 \%)$ & $2(3,4 \%)$ \\
Constipação crônica & $30(50,8 \%)$ & $11(18,6 \%)$ \\
Soiling & $18(50,5 \%)$ & $4(6,8 \%)$ \\
\hline
\end{tabular}


Tabela 4. Eficácia do tratamento 30 dias após a última sessão.

\begin{tabular}{lcccc}
\hline Eficácia do tratamento & $\mathbf{1}^{\mathbf{0}}$ Grau & $\mathbf{2}^{\mathbf{0}}$ Grau & $\mathbf{3}^{\mathbf{0}}$ Grau & Total \\
\hline Cura & $3(60,0 \%)$ & $29(87,9 \%)$ & $16(76,2 \%)$ & $48(81,4 \%)$ \\
Mamilo residual & $2(40,0 \%)$ & $4(12,1 \%)$ & $5(23,8 \%)$ & $11(18,6 \%)$ \\
Total & 5 & 33 & 21 & 59 \\
\hline
\end{tabular}

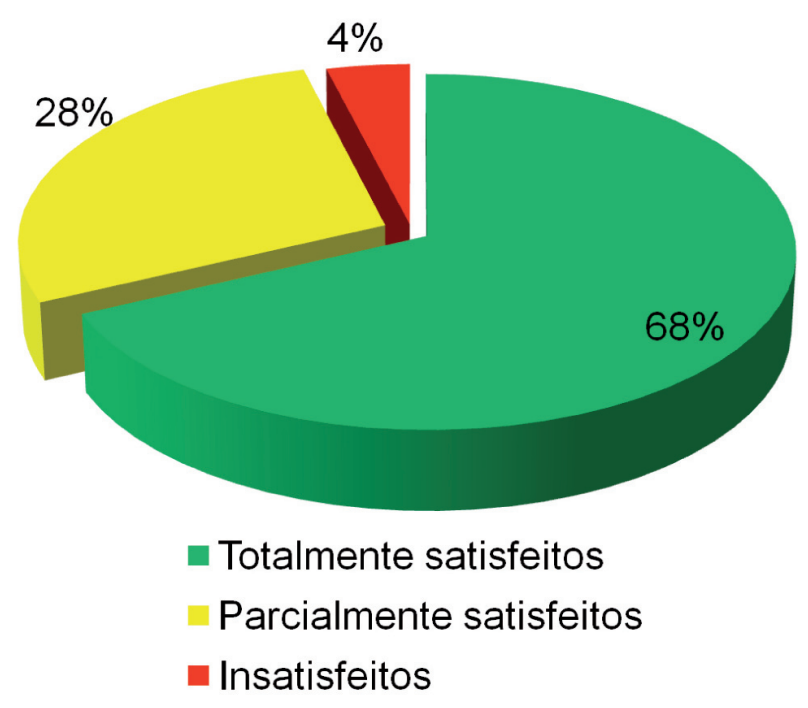

Gráfico 1. Gráfico do grau de satisfação dos pacientes no $30^{\circ}$ dia após o final do tratamento.

são anal de repouso. A etiopatogenia da DH seria decorrente do deslizamento desses coxins por perda da elasticidade dos elementos naturais de sustentação, sob a ação dos fatores desencadeantes do esforço ${ }^{8}$. As hemorroidas são classificadas em internas (quando localizadas na submucosa anorretal acima do ligamento de Parks, recobertas por epitélio mucoso), externas (abaixo do ligamento de Parks, recobertas por pele modificada do canal anal) e mistas (extensões internas e externas $)^{9}$. Nossos achados demográficos corroboram com os da literatura que dizem que a DH incide de forma ligeiramente mais frequente entre as mulheres. Além disso, a média de idade (45,05 anos) também está dentro da faixa etária de maior incidência da $\mathrm{DH}$, que compreende a quarta e quinta décadas de vida9

Os principais sinais e sintomas da DH são: hematoquezia, prolapso da mucosa anal ao ato de defecar, dor anal, ardência anal e prurido anal ${ }^{9,2}$. No nosso estudo, dor anal, prurido anal, prolapso da mucosa anal ao ato de defecar e hematoquezia constituíram-se nos sinais e sintomas mais frequentes da $\mathrm{DH}$, com uma prevalência de $67,8 \%, 59,3 \%, 52,5 \%$ e $50,8 \%$, respectivamente. Além disso, a constipação crônica foi relatada por $51,7 \%$ dos nossos pacientes.

Apesar de a técnica da LE apresentar benefícios em relação ao tratamento cirúrgico da $\mathrm{DH}$, como: simplicidade de execução da técnica, baixo custo, realização ambulatorial e sem o emprego de anestesia, ela não é totalmente isenta de complicações. Tais complicações geralmente são transitórias e, dentre as mais frequentes, podemos citar: dor anal, tenesmo, hematoquezia, sintomas vaso-vagais e retenção urinária. Alguns trabalhos mostram complicações mais graves como sangramentos vultosos que necessitaram de hemotransfusão e abscessos perineais ${ }^{6,10}$. Quando levamos em conta todas as 135 sessões que foram realizadas no nosso trabalho, encontramos que em apenas 23 $(17,1 \%)$ delas não houve relato de dor. Depois da dor, as queixas mais frequentes após as sessões foram, respectivamente: hematoquezia $(45,9 \%)$, dor ao evacuar $(21,5 \%)$, tenesmo $(20,0 \%)$ e tenesmo vesical $(11,1 \%)$. Somente em uma $(0,7 \%)$ sessão tivemos pseudoestrangulamento como complicação. Contudo, o paciente evoluiu bem e pôde continuar o tratamento até o final. Além disso, em 112 (83,0\%) sessões, os pacientes necessitaram ausentar-se de suas atividades laborais apenas no dia em que a sessão foi realizada. Concluímos, portanto, que a LE traz benefícios econômicos que vão além do seu baixo custo de realização.

Como a dor é uma das complicações mais frequentes após a LE, alguns estudos foram feitos com o intuito de descobrir a melhor forma de evitá-la ou minimizá-la ${ }^{11,12}$. Alguns serviços advogam a ligadura de apenas um mamilo por sessão como forma de minimizar a dor ${ }^{13}$. No nosso estudo, para avaliarmos se existiu alguma diferença na morbidade quando ligamos dois mamilos em uma única sessão em vez de apenas um mamilo, separamos um grupo de 40 pacientes. Esses indivíduos tiveram dois mamilos ligados na primeira sessão e um mamilo hemorroidário ligado na segunda sessão. Os resultados mostram que houve uma redução 
de $55,0 \%$ na incidência de dor intensa entre a primeira e a segunda sessão. Além disso, enquanto apenas três $(7,5 \%)$ pacientes não relataram dor na primeira sessão, na segunda este número foi de nove $(22,5 \%)$ pacientes. Não só a intensidade como a duração da dor foi menor na segunda sessão. Na primeira sessão, dentre aqueles que sentiram dor, apenas $29,7 \%$ afirmaram que a dor cessou em menos de 24 horas e em 43,2\% ela durou mais que 48 horas. Já na segunda sessão, esses números foram, respectivamente, $54,8 \%$ e $29,0 \%$. Porém, é prudente questionar a presença de um viés na percepção que cada paciente tem da dor, principalmente após já ter sido submetido a uma sessão de LE. Outras complicações também foram menos comuns na segunda sessão: sintomas vagais $(10,0 \%$ na primeira, contra $2,5 \%$ na segunda), hematoquezia $(57,5 \%$ contra $35,0 \%)$, tenesmo $(25,0 \%$ contra $20,0 \%)$, tenesmo vesical $(15,0 \%$ contra $7,5 \%)$ e dor ao evacuar $(27,5 \%$ contra $25,0 \%)$. Apesar da maior morbidade na segunda sessão, não houve complicações graves o suficiente para impedir a realização de duas ligaduras em uma única sessão. Por isso, acreditamos que o benefício de realizar até duas ligaduras por sessão, diminuindo o tempo de tratamento, reduzindo custos e permitindo que um maior número de pessoas tenha acesso a esse procedimento, supera os riscos de uma morbidade um pouco elevada.

$\mathrm{O}$ tratamento da $\mathrm{DH}$ pelo método da ligadura elástica (LE) tem-se mostrado bastante eficaz quando aplicado a hemorroidas de primeiro, segundo e terceiro graus $^{2}$. Nossos pacientes foram avaliados 30 dias após o término do tratamento. Observamos que a frequência de todos os sinais e sintomas presentes no quadro clínico inicial tiveram uma redução ao final do tratamento. Assim, prurido anal estava presente em dez $(16,9 \%)$ pacientes, ardor anal após a defecação também em dez $(16,9 \%)$, dor anal em cinco $(8,5 \%)$, soiling em quatro $(6,8 \%)$, hematoquezia em dois $(3,4 \%)$ e prolapso da mucosa anal ao ato de defecar também em dois $(3,4 \%)$ pacientes. Esses números, quando comparados com os do quadro clínico inicial, mostram como a LE foi eficiente na diminuição dos sintomas da doença hemorroidária. Além disso, os pacientes sempre eram orientados a manter uma dieta rica em fibras, com o objetivo de melhorar a formação do bolo fecal. Dessa forma, houve redução na prevalência de constipação, estando presente, ao final do tratamento, em apenas 11 $(18,6 \%)$ pacientes. A cura do prolapso hemorroidário, avaliada pela anuscopia 30 dias após a última sessão, foi alcançada em $48(81,4 \%)$ pacientes. A eficácia foi maior nos portadores de DH grau II, apresentando uma taxa de cura de $87,9 \%$, contra $76,2 \%$ na DH grau III. Devemos ressaltar que dos $11(18,6 \%)$ pacientes que apresentaram mamilo residual, apenas uma paciente não foi encaminhada para LE adicional, por ter sido diagnosticado gravidez nos 30 dias entre a última sessão e a consulta de revisão. Os pacientes encaminhados a mais uma sessão de LE provavelmente estão curados do prolapso hemorroidário. Porém, foge ao escopo deste trabalho avaliar a resposta que esses dez pacientes tiveram à LE adicional. Também tivemos um paciente, portador de DH grau III, que necessitou realizar seis sessões. Este, ao ser reavaliado 30 dias após a última sessão, apresentou-se curado do prolapso hemorroidário e com remissão de todos os sintomas.

Ao serem questionados quanto à satisfação ao final do tratamento, 40 pacientes $(67,8 \%)$ afirmaram estar completamente satisfeitos, 17 pacientes $(28,8 \%)$ estavam parcialmente satisfeitos e dois $(3,4 \%)$ pacientes disseram estar insatisfeitos com o tratamento. Devemos ressaltar que muitos pacientes não tiveram satisfação plena devido à permanência de plicomas residuais. Esse dado foi obtido a partir da conversa com os pacientes, mas não era objetivo deste trabalho quantificá-lo.

\section{CONCLUSÕES}

A DH foi mais frequente entre as mulheres (56,7\% dos pacientes). A idade variou entre 19 e 70 anos, com uma média de 45,08 anos.

Os sintomas mais prevalentes da DH foram: dor anal, prurido anal, prolapso da mucosa anal ao ato de defecar e hematoquezia. As complicações mais frequentes da LE foram, respectivamente: dor anal, hematoquezia, dor ao evacuar, tenesmo e tenesmo vesical.

O tempo médio de afastamento das atividades laborais foi de 1,6 dias por sessão. Ao comparar sessões em que apenas uma ligadura foi realizada com aquelas em que se realizaram duas ligaduras, encontramos uma morbidade maior no último caso.

A análise dos dados nos permite afirmar que a ligadura elástica é um procedimento eficaz para o tratamento da DH (eficácia global de $81,4 \%$, eficácia de $87,9 \%$ nos portadores de DH grau II e de $76,2 \%$ nos que tinham DH grau III).

Houve um alto grau de satisfação com o procedimento. 
ABSTRACT: Rubber band ligation (RB) is considered a minimally invasive method for the treatment of hemorrhoidal disease (HD) and has advantages in relation to hemorrhoidectomy whereby: simplicity of execution, outpatient realization and no need of anesthesia. It is an effective method, especially in the HD grade II. However, shows complications, and the most frequents are: anal pain, tenesmus, hematochezia and urinary retention. Some studies have shown severe complications as major bleeding that needs blood transfusion. Thus, this study evaluated the effectiveness and the morbidity of the treatment of HD by the method of RB. It was a prospective study with 59 patients. Five $(8,5 \%)$ patients had HD graded in the first degree, $33(55,9 \%)$ in the second degree and $21(35,6 \%)$ in the third. All patients were submitted to at least two sessions. In the 135 sessions performed, we found: hematochezia in 62 (45.9\%), severe pain in $39(28.9 \%)$, vagal symptoms in $10(7.4 \%)$ and pseudostrangulation in $1(0.7 \%)$ session. The cure rate of hemorrhoidal prolapse among patients with HD grade II was $87.9 \%$ and among those with HD grade III, 76.2\%. The treatment of HD by the method of $R B$ proved to be safe and with good cure rate.

Keywords: hemorrhoid; rubber band ligation; complications.

\section{REFERÊNCIAS}

1. Keighley MRB, Williams NS. Cirurgia do ânus, reto e colo. São Paulo: Manole; 1998.

2. El Nakeeb AM, Fikry AA, Omar WH, Fouda EM, El Metwally TA, Ghazy HE, et al. Rubber band ligation for 750 cases of symptomatic hemorrhoids out of 2200 cases. World J Gastroenterol 2008;14(42):6525-30.

3. Murie JA, MacKenzie I, Sim AJ. Comparison of rubber band ligation and haemorrhoidectomy for second and third degree haemorrhoids: a prospective clinical trial. Br J Surg 1980;67(11):786-8.

4. Kumar N, Paulvannan S, Billings PJ. Rubber band ligation of haemorrhoids in the out-patient clinic. Ann R Coll Surg Engl 2002;84(3):172-4.

5. Bernal JC, Enguix M, García JL, Romero JG, Peris RT. Ligadura con banda elástica de las hemorroides en una Unidad de Coloproctología: Estudio prospectivo. Rev esp enferm dig 2005;97(1):38-45.

6. Moreira CAX. Tratamento de hemorróidas pelo método da ligadura elástica. Rev bras Coloproct 1981;1(2):27-31.

7. Watson NFS, Liptrott S, Maxwell-Armstrong CA. A prospective audit of early pain and patient satisfaction following out-patient band ligation of haemorrhoids. Ann R Coll Surg Engl 2006;88(3):275-9.
8. Haas PA, Fox TA Jr, Hass JP. The pathogenesis of Hemorrhoids. Dis Colon and Rectum 1984; 27(7):442-50.

9. Cruz GMG, Ferreira RMRS, Neves PM. Doença hemorroidária - Aspectos epidemiológicos e diagnósticos de 9.289 pacientes portadores de doença hemorroidária. Rev bras Coloproct 2006;26(1):6-23.

10. Sim HL, Tan KY, Poon PL, Cheng A, Mak K. Life-threatening perineal sepsis after rubber band ligation of haemorrhoids. Tech Coloproctol 2009;13(2):161-4.

11. Costa e Silva IT, Chamhic JE, Novo NF, Juliano Y, Goldenberg S. Freqüência de dor após a ligadura elástica de hemorróidas: estudo prospectivo e randomizado. Rev bras Coloproct 1990;10(4):134-8.

12. Fernandes RHO, Canali C, Bertuol I, Longhi JA. Uso de lidocaína spray em ligaduras elásticas de hemorróidas Estudo prospectivo. Rev bras Coloproct 2003;23(3):183-6.

13. Stern H, McLeod R, Cohen Z, Ross T. Ambulatory procedures in anorectal surgery. Adv Surg 1987;20(1): 217-44.

Endereço para correspondência:

Juvenal da Rocha Torres Neto

Rua Ananias Azevedo, 100, ap. 902 - Praia 13 de Julho

CEP: 49020-080 - Aracaju (SE), Brasil

E-mail: jtorres@infonet.com.br 